\title{
Assessment of different methods for diagnosis of Group B streptococci during pregnancy
}

\begin{abstract}
Objectives: To compare the different diagnostic techniques used to detect GBS colonization in pregnant women in late third trimester after thirty five weeks and to detect the frequency of GBS colonization among a sample of pregnant Egyptian women.

Patients and methods: Vaginal swabs from the lower third of vagina were collected from 100 pregnant women in the late third trimester. Isolation of the organism by culture on selective media and confirmation by latex agglutination test and detection of CAMP factor by conventional PCR were compared. GBS isolates were tested by double disk diffusion method and D-zone test simultaneously for susceptibility to erythromycin and clindamycin and inducible clindamycin resistance for intrapartum antibiotic prophylaxis (IAP).
\end{abstract}

Results: 25 participants $(25 \%)$ were positive for GBS by culture in Lim broth with subculture onto TSA supplemented with 5\% defibrinated sheep blood, while 75 participants (75\%) were negative. Of the $25 \mathrm{GBS}$ isolates, 19 (76\%) were sensitive to erythromycin, $3(12 \%)$ were intermediate and $3(12 \%)$ were resistant. Of the 25 GBS isolates, $15(60 \%)$ were sensitive to clindamycin, $2(8 \%)$ were intermediate and $8(32 \%)$ were resistant. Fourteen isolates $(56 \%)$ were sensitive to both erythromycin and clindamycin whereas 3 $(12 \%)$ were resistant to both (cMLSB). Latex agglutination test for GBS detection from the 24 hours incubated Lim broth was positive in 25 cases $(25 \%)$. GBS was detected in 9 cases $(9 \%)$ by the conventional PCR assay done directly from vaginal swabs specimens. Sensitivity, specificity, PPV and NPV for latex agglutination from the inoculated broth and PCR assay are $100 \%, 100 \%, 100 \%, 100 \%$ and $36 \%, 100 \%, 100 \%, 82.4 \%$ respectively. Latex agglutination test from the inoculated broth showed a statistically significant perfect agreement (100.0\%) with culture with Kappa value 1.0 and 95\% CI (1.0 - 1.0). PCR assay also showed a statistically significant but moderate agreement $(84.0 \%)$ with culture with Kappa value 0.458 and $95 \%$ CI $(0.253-0.662)$.

Conclusion: Detection of GBS colonization by latex agglutination test from incubated selective broth directly is comparable to the gold standard (culture) as regards accuracy. PCR offers a rapid and highly specific method for detection of GBS colonization especially in intrapartum settings for administration of IAP in non-screened pregnant females; however, sensitivity is low resulting in a low NPV.

Keywords: GBS, latex agglutination, PCR, D-zone test
Volume II Issue 5 - 2020

\author{
Ahmed SSA Rashwan \\ Department of Obstetrics and Gynecology, Faculty of Medicine, \\ Cairo University, Egypt \\ Correspondence: Ahmed Rashwan, MD, Department \\ of Obstetrics and Gynecology, Faculty of Medicine, Cairo \\ University, Villa 36 A, Maadi Heights Compound-Tabarak City, \\ Egypt, Tel +201002592250, Email ahmedrashwan I8I@gmail.com
}

Received: August 24, 2020 | Published: September 07, 2020

\section{Introduction}

Neonatal morbidity and mortality occur significantly in pregnancies associated by GBS. ${ }^{1}$ So, working hard to detect and prevent this microbial colonization is an important target. ${ }^{2}$

Early-onset disease (EOD) occurs within few hours after delivery up to 6 days of age. Late-onset disease (LOD) starts from 7 days of age, and usually leads to fever or meningitis, but is less often fatal compared to early infection. ${ }^{3}$

Currently, recommendations are focusing on maternal GBS screening. ${ }^{4}$ Screening of pregnant females for GBS colonization and intrapartum antibiotic prophylaxis is very important for prophylaxis against early-onset GBS disease. $^{5}$

CDC recommended inoculation of vaginal or vagino-rectal swabs into a selective broth for enrichment and further subculture onto solid media (the gold standard). ${ }^{6}$

The culture method has a slow turnaround time ( 36 to $72 \mathrm{~h}$ ) before results can be issued. In addition to being time consuming, it requires an experienced technician to identify the suspected colonies, which are not always beta-hemolytic. ${ }^{7}$

Polymerase chain reaction (PCR) and optical immunoassay (OIA) are candidates for rapid near patient intrapartum GBS testing to determine whether women in labor are colonized with GBS or not. ${ }^{8,9}$

\section{Materials and methods}

This was a cross-sectional study conducted to compare different diagnostic techniques for GBS and to detect the frequency of GBS vaginal colonization in a sample of Egyptian pregnant women to reduce the possibility of neonatal diseases due to vertical transmission according to CDC (2010). ${ }^{5}$ One hundred pregnant women in the late third trimester (35-37 weeks of gestation) coming for antenatal care in outpatient clinic of Obstetrics and Gynecology Department, Kasr Al-Ainy University Hospitals during the period from April 2018 to October 2018, were included in the study.

There were no exclusion criteria except for receiving any systemic or local vaginal antimicrobial agents during the preceding 2 weeks. 
After an informed consent was taken from the participants, they were subjected to: Full history taking including name, age and obstetric history (e.g. number of pregnancies, history of previous preterm labour, history of previous premature rupture of membranes (PROM), history of previous neonatal sepsis and number of normal vaginal deliveries).

Also, a phone number (if available) was taken from the subjects to report the result of a positive culture of GBS. GBS carriers were advised to take antibiotic therapy for elimination of GBS vaginal colonization.

After history taking, the following was done according to CDC $(2010)^{5}$ :

A. Lower vaginal swab specimens were obtained.

B. Testing for the presence of GBS colonization was done using:

i. Inoculation into Lim broth with subculture on tryptone soy agar (TSA) supplemented with 5\% defibrinated sheep blood. This is considered to be the gold standard for detection of GBS colonization.

ii. Latex agglutination test for the incubated Lim broth.

iii. Conventional PCR technique.

C. In addition, GBS isolates recovered from vaginal swabs on TSA were tested for inducible clindamycin resistance by the D-zone test.

\section{Collection of lower vaginal swabs}

After taking an informed consent, 3 lower vaginal swabs were collected from each pregnant woman. Sterile cotton-tipped swabs were used to collect specimens by introducing the swab 1-2 inches beyond the vaginal vestibule and rotating it against the vaginal wall. All participants refused to have vaginal-anal swabbing despite the possible higher yield of GBS as in CDC (2010), ${ }^{5}$ so only lower vaginal swabs were taken.

\section{Detection of GBS by culture}

Inoculation into Lim broth (bioMérieux, France): One of the collected swabs was used for the isolation of GBS by immediate inoculation at the bed-side into a tube of Lim broth. The Lim broth tubes were used as both transport and enrichment media. The tubes were closed tightly after proper labelling (data included name and number of the subject, date and time of collection), stored at room temperature during transport to the laboratory according to the manufacturer's instructions and incubated at $37^{\circ} \mathrm{C}$ aerobically for 18 $24 \mathrm{hrs}$ in the laboratory maximally within $3 \mathrm{hrs}$ of collection.

\section{Processing of the inoculated lim broth}

After incubation, each swab was discarded. The inoculated broth was used for: subculture onto TSA and latex agglutination test.

Subculture onto TSA: According to Smith et al., ${ }^{10} 10 \mu 1$ of the inoculated broth were subcultured onto TSA plates supplemented with $5 \%$ defibrinated sheep blood and incubated at $37^{\circ} \mathrm{C}$ in $5-10 \% \mathrm{CO} 2$ for 18-24hrs. The inoculated plates were examined for GBS growth on the next day. Suspicious colonies are small about $1 \mathrm{~mm}$ in diameter, soft, convex, moist, regular, usually grey white or colourless, usually shiny or dry colonies may be surrounded with narrow hazy zone of beta-hemolysis or non-hemolytic. Any colonies revealing catalasenegative, Gram-positive cocci arranged in short chains or pairs were confirmed to be GBS by latex agglutination test. Plates negative for GBS after 18-24hrs of incubation were re-incubated and re-examined on day 4 (after $48 \mathrm{hrs}$ of incubation) before being discarded as negative. According to Clinical and Laboratory Standards Institute (2013), ${ }^{11}$ GBS isolates were tested for inducible clindamycin resistance using the D-zone test. According to Ke et al. ${ }^{12}$ and Uh et al., ${ }^{13}$ all GBS isolates were frozen at $-80^{\circ} \mathrm{C}$ in brain-heart infusion broth containing $20 \%$ glycerol.

Latex agglutination test: This test was done according to manufacturers' directions for: testing the inoculated Lim broth for GBS and confirmation of suspected GBS colonies on TSA using Slidex Strepto Plus B Kit (bioMerieux, France). In case of inoculated LIM broth testing: $15 \mu \mathrm{l}$ of the inoculated broth were added to the extraction enzyme according to Wali et al. ${ }^{14}$

Testing of GBS isolates for inducible clindamycin resistance using the D-Zone test: D-zone test using double-disk diffusion method was done for the different GBS isolates to identify those that are erythromycin-resistant and clindamycin-susceptible, yet have inducible resistance to clindamycin. It was performed according to Clinical and Laboratory Standards Institute. ${ }^{11}$

Detection of GBS by conventional PCR: Vaginal swabs intended for PCR testing were stored dry at $-80^{\circ} \mathrm{C}$ for $1-3$ months till further processing. The GBS reference strain (ATCC 13813) was obtained from the Mircens, Faculty of Agriculture, Ein Shams University. It was used as a positive control. It was preserved in BHI broth tubes with $20 \%$ glycerol and stored at $-80^{\circ} \mathrm{C}$.

Preparation of genomic DNA from the reference GBS strain: After thawing, subculture of the GBS reference strain on TSA and incubation in candle jar overnight was done. DNA extraction from the obtained GBS growth was done using the boiling method according to Fang and Hedin. ${ }^{15}$

DNA extraction from the vaginal swabs: This was done using Genekam DNA Isolation Kit (Genekam Biotechnology AG, Germany). DNA extraction was done according to the manufacturer's instructions using the buccal swab protocol.

\section{Amplification}

Primers: According to Ke et al., ${ }^{12}$ Bergeron et al. ${ }^{16}$ and Rallu et al., ${ }^{17}$ PCR amplification of cfb gene (encoding the CAMP factor) present in virtually every GBS isolate was performed using Sag 59 and Sag 190 primers. The GBS-specific primers amplify a fragment of $153 \mathrm{bp}$.

Amplification protocol: The protocol adopted for amplification of $\mathrm{cfb}$ gene using PCR master mix (Jena Bioscience GmbH, Germany) was a modification of that described by Ke et al., ${ }^{12}$ Bergeron et al. ${ }^{16}$ and Rallu et al. ${ }^{17}$ A positive control was included in each run and a negative control.

The amplification was performed in Biometra T Personal thermal cycler under the following thermal cycling protocol: An initial denaturation step at $94^{\circ} \mathrm{C}$ for 5 minutes followed by 45 cycles of amplifications, each consisted of: denaturation at $94^{\circ} \mathrm{C}$ for 30 seconds, annealing at $52^{\circ} \mathrm{C}$ for 30 seconds, extension at $72^{\circ} \mathrm{C}$ for 30 seconds followed by final extension at $72^{\circ} \mathrm{C}$ for 10 minutes.

Detection of amplified product by Gel Electrophoresis: Amplicons (10 $\mu 1$ of the amplified reaction mixture) were electrophoresed on 2\% agarose gel (Vivantis) in 1x TAE (Tris acetate EDTA) buffer (Biobasic). Ethidium bromide ( $3 \mu 1$ of $60 \mathrm{mg} / \mathrm{ml}$ solution) was added during preparation of the gel $(75 \mathrm{ml})$ after cooling down. 
Electrophoresis was done at 120volts for 45minutes. Gels were visualized and photographed under ultraviolet illumination using biometra TI-1 Gel Documentation System (biometra). Amplicons were compared to a molecular weight marker (100-1000bp) (Bioron). The entire process of GBS detection by conventional PCR required a minimum of $6 \mathrm{hrs}$

Statistical analysis: All collected questionnaires were revised for completeness and consistency. Pre-coded data were entered on the computer using "Microsoft Office Excel Software" program (2010) for windows. Data were then transferred to the Statistical Package for the Social Sciences (SPSS) version 24 to be statistically analysed.

Data were summarized using mean, and standard deviation for quantitative variables and frequency and percentage for qualitative ones.

Comparison between groups was performed using independent sample t-test for quantitative variables and Chi square or Fissure exact test for qualitative ones.

$\mathrm{P}$ values less than 0.05 were considered statistically significant, and less than 0.01 were considered highly significant.

\section{Results}

One hundred pregnant females, in their third trimester (35-37 weeks of gestation), coming for antenatal care were included in this study. $25(25 \%)$ participants were positive for GBS colonization using culture (the gold standard), while 75 (75\%) participants were negative.

\section{Data of the participants}

Age of the participants: Mean age of the positive cases was $26.2 \pm 4.8$ years while mean age of the negative cases was $27.9 \pm 5.1$ years with no statistically significant difference in the age between positive and negative cases $(\mathrm{P}$-value $=0.2$ ). It has been shown that the highest frequency occurred within the age group 25-30 years while lower frequencies occurred in the other two age groups with no statistically significant difference within each age group.

Parity of the participants: Mean parity of positive cases was $1.4 \pm 1.2$ children while mean parity of negative cases was $1.8 \pm 1.4$ children with no statistically significant difference $(p$-value $=0.3$ ). There is no statistically significant relation between GBS colonization \& the number of previous pregnancies.

Neonatal sepsis in previous pregnancies: Sepsis occurred in previous pregnancies in neonates of two participants only $(2 \%)$. None of these two participants was positive for GBS colonization. There was no statistically significant difference between positive and negative cases as regards the occurrence of neonatal sepsis in previous pregnancies ( $\mathrm{p}$-value $=1$ ).

PROM in previous pregnancies: Twenty four $(24 \%)$ of the cases had history of PROM in previous pregnancies; among them, 4 cases were colonized with GBS (16\% of the positive cases) and 20 were not colonized with GBS (26.7\% of the negative cases). There was no statistically significant difference between positive and negative cases as regards the occurrence of PROM in previous pregnancies ( $\mathrm{p}$-value $=0.4)$

Results of culture for GBS: In this study, 25 participants (25\%) were positive for GBS by culture in Lim broth with subculture onto TSA supplemented with 5\% defibrinated sheep blood, while 75 participants
(75\%) were negative. The same results were obtained after both $24 \&$ $48 \mathrm{hrs}$ of incubation. Results of the culture were available after 48 to $72 \mathrm{hrs}$.

Results of antibiotic susceptibility testing: GBS isolates were tested for their susceptibility to erythromycin and clindamycin by disc diffusion method, in addition to testing for clindamycin inducible resistance by the D-zone test. ${ }^{11}$

Susceptibility to erythromycin: Of the 25 GBS isolates, $19(76 \%)$ were sensitive to erythromycin, $3(12 \%)$ were intermediate and 3 $(12 \%)$ were resistant.

Susceptibility to clindamycin: Of the 25 GBS isolates, $15(60 \%)$ were sensitive to clindamycin, $2(8 \%)$ were intermediate and $8(32 \%)$ were resistant.

Fourteen isolates $(56 \%)$ were sensitive to both erythromycin and clindamycin whereas $3(12 \%)$ were resistant to both (cMLSB). It was noticed that none of the isolates was resistant to erythromycin and sensitive to clindamycin. Table 1 summarizes the different patterns of susceptibility of GBS isolates to erythromycin and clindamycin.

Table I The different patterns of susceptibility of GBS isolates

\begin{tabular}{|c|c|c|c|c|c|c|}
\hline \multicolumn{3}{|c|}{ Erythromycin } & \multicolumn{3}{|c|}{ Clindamycin } & $\begin{array}{l}\% \text { of GBS isolates } \\
(n=25)\end{array}$ \\
\hline S & I & $\mathrm{R}$ & $\mathrm{S}$ & I & $\mathrm{R}$ & \\
\hline \multirow[t]{2}{*}{$\sqrt{ }$} & & & $\sqrt{ }$ & & & $56 \%(14)$ \\
\hline & & $\sqrt{ }$ & & & $\sqrt{ }$ & $12 \%$ (3) (cMLSB) \\
\hline$\sqrt{ }$ & & & & & $\sqrt{ }$ & $12 \%(3)$ \\
\hline \multirow[t]{3}{*}{$\sqrt{ }$} & & & & $\sqrt{ }$ & & $8 \%(2)$ \\
\hline & $\sqrt{ }$ & & & & $\sqrt{ }$ & $8 \%(2)$ \\
\hline & $\sqrt{ }$ & & $\sqrt{ }$ & & & $4 \%(I)$ \\
\hline
\end{tabular}

Inducible clindamycin resistance of the GBS isolates: Inducible clindamycin resistance is detectable by the D-zone test in GBS isolates that are resistant to erythromycin but sensitive to clindamycin. As none of the isolates showed this pattern of susceptibility, D-zone test was not applicable to GBS isolates in this study.

Results of latex agglutination test for detection of GBS from the inoculated Lim broth: Latex agglutination test for detection of GBS from the $24 \mathrm{hrs}$ incubated Lim broth was positive for GBS in 25 cases $(25 \%)$.The same cases were also positive for GBS after subculture of incubated Lim broth onto TSA. The organism was not detected by latex agglutination test in any of the samples that proved later to be negative by culture. Results of the latex agglutination test from the Lim broth were available after $24 \mathrm{hrs}$.

\section{Discussion}

Approximately $10 \%-30 \%$ of pregnant women are colonized with GBS in the vagina or rectum. GBS colonization during pregnancy can be transient, intermittent, or persistent. ${ }^{18}$

The exact site swabbed influences the results. In 2010, CDC recommended the use of a single swab of the lower vagina and the anorectum to be transported to the laboratory in a selective broth medium and subcultured onto a selective solid medium for optimal recovery of GBS. 
Among 112 pregnant women, $29.5 \%$ were identified as carriers of GBS on the basis of the results of culture of combined vaginal and anal specimens, as compared with $17.9 \%$ of vaginal specimens and $26.8 \%$ of anal specimens. ${ }^{16}$

In 2009, El Aila et al. also conducted a study to compare the difference in GBS recovery rate from combined vaginal-anal, vaginal and anal swabs for 150 pregnant women. GBS were detected in 36 women. Of these, 19 harbored GBS in both rectum and vagina, 9 only in the vagina and 8 exclusively in the rectum. ${ }^{19}$

Therefore, to perform the present study it would have been preferable to take a combined distal vaginal and anorectal swab, but the studied group refused anorectal swabbing and specimens taken only from the vagina were available for GBS isolation.

Kulkarni et al. ${ }^{20}$ compared selective broth medium and Stuart transport medium as transport systems used for GBS isolation. Selective broth medium was the most effective as it inhibits the normal vaginal flora without any inhibitory effect on isolation of GBS. Moreover, it acts as both transport as well as enrichment medium for GBS. Therefore, Lim broth, being one of the selective transport media, was used as both transport and enrichment medium in the present study. ${ }^{20}$

Among the 100 cases tested in the present study, 25\% were positive for GBS using the gold standard (culture on solid media) as approved by CDC in 2010. Results did not differ with extended incubation of the plates for an additional 24 hours.

A systematic review on the prevalence of maternal GBS colonization in European countries revealed that GBS vaginal colonization rates ranged from $6.5 \%$ to $36 \%$, with one-third of the studies reporting rates of $20 \%$ or greater. There was a variation in the carriage rates: Eastern Europe $19.7 \%$ to $29.3 \%$, Western Europe $11 \%$ to $21 \%$, Scandinavia $24.3 \%$ to $36 \%$ and Southern Europe $6.5 \%$ to $32 \% .^{21}$

In 2007, Kovavisarach et al. ${ }^{22}$ stated that one of the risk factors for GBS colonization in pregnant women was older maternal age. ${ }^{22}$ However, in the present study no statistically significant difference was found in the age between positive and negative cases which agrees with the studies done by Atkins et al., ${ }^{23}$ Jahromi et al. ${ }^{24}$ and Dechen et al. ${ }^{25}$

In the present study, no significant difference was found in the carriage rate of GBS between cases of high parity and cases of low parity. This is similar to the results of Atkins et al., ${ }^{23}$ Costa et al. ${ }^{26}$ and Dechen et al. ${ }^{25}$ who found that the relation between gravidity and GBS culture positivity was statistically insignificant.

In the present study, there was no statistically significant difference between positive and negative cases as regards the occurrence of neonatal sepsis or the occurrence of PROM in previous pregnancies.

In contrast to our findings, Jahromi et al. ${ }^{24}$ found that preterm birth, prolonged rupture of membranes, and preterm premature rupture of membranes were more common among GBS colonized mothers. Dechen et al. ${ }^{25}$ also reported that GBS infection among pregnant women was significantly related with the gestational age $(<36$ weeks), PROM and preterm labor.

In this study, 25 cases $(25 \%)$ were positive for GBS by culture in Lim broth followed by subculture onto TSA with $5 \%$ defibrinated sheep blood. No direct plating on blood agar was done. The total time required was $48 \mathrm{~h}$ as examination of the plates after $72 \mathrm{~h}$ revealed no difference in the results.

Many studies supported the importance of using Lim broth followed by subculture for detection of GBS such as Kulkarni et al. ${ }^{20}$ and El Sayed et al. ${ }^{27}$ as unfortunately, direct plating of swabs yields false-negative culture results in as many as $50 \%$ of GBS-colonized women. ${ }^{28}$

Moreover, in their study, El Aila et al. ${ }^{19}$ compared 3 culture techniques. Of 55 samples from which GBS was isolated, 55 were positive by Lim broth enrichment with subculture on Granada agar, 45 by Lim broth enrichment with subculture on Columbia CNA agar and 22 by direct inoculation on Columbia CNA agar.

In order to decrease the time for detection of GBS using culture, special media (e.g. media of Islam's and Granada agar media) have been suggested. They have the great advantage of being able to identify GBS growth sometimes within 4-18 h due to the production of carotenoid orange pigment by the organism. However, not all GBS strains are able to produce this carotenoid pigment which, curiously, was found to be associated with haemolysin biosynthesis. ${ }^{29}$ In addition, unfortunately both Granada and Islam's media have a limited shelf-life. ${ }^{30}$

In this study inoculation of the swabs into Lim broth for $18 \mathrm{~h}$ and performing latex agglutination test for GBS identification was compared with the gold standard. The rate of GBS isolation was similar to standard culture $(25 \%)$ whereas total time required was shortened to 20h. The sensitivity, specificity, PPV and NPV for latex agglutination from incubated Lim broth were all 100\%. Agreement between latex agglutination from incubated Lim broth and culture was found to be a statistically significant perfect agreement (100\%).

In 2007, Wali et al. reported that GBS carriage in the vagina of pregnant Egyptian women at 35-37 weeks of gestation was 15.7\% using Lim broth incubation followed by latex agglutination test. Therefore, they recommended the use of this method even over culture as it showed the highest rate of detection and the total time required was $24 \mathrm{~h}$ (culture recorded a carriage rate of only 14\%). Latex agglutination from incubated Lim broth provides many advantages: it has a good sensitivity, a reduction in the workload, cost and a TTR of $24 \mathrm{~h}$. In addition, professional personnel for GBS detection from the plates are not needed. These findings are in accordance with the present study. ${ }^{14}$.

The culture-based screening methods may require up to $72 \mathrm{~h}$ before a final result is achieved. Efforts to minimize TTR have led to the development of molecular-based methods, such as real time-based PCR and "peptide nucleic acid fluorescence in situ hybridization" (PNA FISH) ${ }^{31}$

In the present study, GBS was detected in only $9 \%$ by the conventional PCR assay (targeting $c f b$ gene) done directly from vaginal swabs specimens (total time required was $6 \mathrm{~h}$ ); all of the positive cases by PCR were positive by culture. This indicates that molecular methods are more rapid and highly specific but have questioned sensitivity in comparison with culture. The sensitivity, specificity, PPV and NPV for PCR were 36\%, 100\%, 100\%, 82.4\% respectively. Agreement between PCR and culture was found to be a statistically significant but moderate agreement (84\%).

Different studies reported a wide range of variation in the results of PCR as a method for detection of GBS from vaginal specimens, 
especially as regards sensitivity of the assay. For example, Bergeron et al. ${ }^{16}$ developed 2 PCR assays (conventional and real-time) for GBS detection. They compared these PCR assays with the standard culture. Time required to obtain results was 30-45 minutes for the real-time assay, 100 minutes for the conventional and at least 36 hours for culture. As compared with culture, the sensitivity of both assays was $97 \%$ and NPV was $98 \%$. The specificity and PPV of the PCR assays were both $100 \%$. Ke et al. stated that real-time PCR assay was comparable to conventional PCR assay in terms of sensitivity and specificity, but more rapid, requiring only $30 \mathrm{~min}$. versus $100 \mathrm{~min} .{ }^{12}$

Bergh et al. ${ }^{32}$ constructed a real-time PCR assay for detection of GBS using the sip gene. Its performance was tested on vaginal specimens and compared to culture. Culture was positive in $25 \%$ of studied cases while PCR was positive in $32 \%$. The authors concluded that real-time PCR was sensitive, rapid and TTR was $<2 \mathrm{~h}$. Due to its rapidity, it harbors the potential for intrapartum GBS detection.

Samples taken in Iran were tested by culture and by PCR targeting $c f b$ gene. Culture identified $9.3 \%$ as carriers of GBS, compared to $11.2 \%$ by PCR. ${ }^{7}$ The sensitivity and NPV of the PCR were $100 \%$ compared with culture. The specificity and PPV of the PCR were $98 \%$ and $100 \%$, respectively. TTR was $3 \mathrm{~h}$ for PCR and at least $36 \mathrm{~h}$ for culture.

Other studies demonstrated a lower sensitivity for PCR. For example, Atkins et al. ${ }^{23}$ investigated recto-vaginal samples for GBS detection by culture and real-time PCR. Sensitivity of PCR was $86.8 \%$, specificity was $95.2 \%$, PPV was $88.1 \%$ and NPV was $94.6 \%$. They concluded that the false-negative results of the real-time PCR (13.2\%) prohibit its use for standard GBS screening. A 77\% sensitivity of the BD GeneOhm StrepB PCR assay done directly from swabs was reported by Smith et al. ${ }^{33}$ when compared to culture as a gold standard.

Even lower sensitivities for PCR were recorded by other investigators. For example, the sensitivity of direct swab PCR was found to be $59 \%$ as reported by Block et al. ${ }^{34}$ and $52 \%$ in the study by Munson et al. ${ }^{35}$

The "false negatives" are most likely a result of sampling error, low levels of the organism in the sample, overgrowth of the normal vaginal flora or the absence of the gene tested. In addition, DNA amplification is sensitive to numerous inhibitors which can be included in the DNA extract from the clinical material. ${ }^{36}$ Moreover, isolation of nucleic acids from such a material results in the presence of a significant amount of human DNA which may interact with bacterial DNA; this will evidently lower PCR sensitivity. In the study of Bergeron et al., ${ }^{16}$ DNA extraction was done from the transport medium used (Stuart's) and tested within $24 \mathrm{~h}$ after collection. Any of these causes might have been responsible for the repeatedly negative results following retesting of culture positive samples by PCR in the present study.

Surveillance has shown that GBS remains universally susceptible to penicillins and glycopeptides such as vancomycin. On the other hand, Kimura et al. ${ }^{37}$ reported the finding of clinical GBS isolates with reduced penicillin susceptibility and limited studies reported elevated penicillin MIC level for GBS. ${ }^{38}$

In the present study, erythromycin and clindamycin susceptibility pattern was determined by disc diffusion method and inducible clindamycin resistance was simultaneously detected by the D-zone test.

Of the 25 GBS isolates, 19 (76\%) were sensitive to erythromycin, $3(12 \%)$ were intermediate and $3(12 \%)$ were resistant. As regards clindamycin, $15(60 \%)$ were sensitive, $2(8 \%)$ were intermediate and $8(32 \%)$ were resistant. Fourteen isolates $(56 \%)$ were sensitive to both antibiotics and $3(12 \%)$ were resistant to both $\left(\mathrm{cMLS}_{\mathrm{B}}\right)$. The remaining isolates showed other different patterns of susceptibility. None of the isolates was resistant to erythromycin and sensitive to clindamycin; therefore, detection of inducible clindamycin resistance by the D-zone test was not an option in this study though it was planned to be tested for.

GBS resistance rates to erythromycin and clindamycin differ according to different regions of the world, being relatively low in northern Europe, ${ }^{39}$ high in southern Europe, ${ }^{40}$ and highest in the Far East. ${ }^{41}$ In New Zealand, $15 \%$ and $7.5 \%$ of GBS isolates were resistant to clindamycin and erythromycin, respectively. ${ }^{42}$

In contrast to our results, a previous study showed a higher rate of resistance to clindamycin than to erythromycin..$^{43}$ In the Turkish study (2004), all isolates were susceptible to penicillin, while $22.4 \%$ were resistant to erythromycin comparable to our results. ${ }^{44}$

From this study, it was concluded that GBS colonization is common among pregnant women in Egypt with a frequency similar to that of other countries either the developed or the developing. Detection of GBS colonization by performing latex agglutination test from incubated selective broth directly is comparable to the gold standard (culture in selective broth with subculture on solid media) as regards accuracy. In addition, it decreases the time to obtain results (TTR $18 \mathrm{~h}$ versus $48 \mathrm{~h}$ ), costs, workload and it does not require experienced laboratory personnel. PCR offers a rapid (within hours) and highly specific method for detection of GBS colonization especially in intrapartum settings for administration of IAP in nonscreened pregnant females; however, sensitivity is low resulting in a low NPV. GBS resistance to erythromycin and clindamycin is not uncommon, which may denote that antimicrobial resistance in GBS is a serious and an increasing problem.

\section{Conclusion}

Detection of GBS colonization by latex agglutination test from incubated selective broth directly is comparable to the gold standard (culture) as regards accuracy. PCR offers a rapid and highly specific method for detection of GBS colonization especially in intrapartum settings for administration of IAP in non-screened pregnant females; however, sensitivity is low resulting in a low NPV.

\section{Acknowledgments}

None.

\section{Funding}

None

\section{Conflicts of interest}

The authors confirm the absence of any other conflict of interest that needs to be reported.

\section{References}

1. Seale A, Bianchi-Jassir F, Russell N. Estimates of the burden of group B streptococcal disease worldwide for pregnant women, stillbirths and children. Clinical Infectious Diseases. 2017;65(Suppl 2):S200-S219.

2. Ahmadzia H.K, Heine RP. Diagnosis and management of group B streptococcus in pregnancy. Obstetrics and Gynecology Clinics of North America. 2014;41(4):629-647. 
3. Heath PT, Jardine LA. Neonatal infections: group B streptococcus. Clin Evid. 2010

4. American College of Obstetricians and Gynecologists Committee on Obstetric Practice. ACOG Committee Opinion no. 485: prevention of early-onset group B streptococcal disease in newborns. Obstetrics \& Gynecology. 2011;117(4):1019-1027.

5. Jennifer RV, Lesley McGee, Stephanie JS, et al. Prevention of perinatal group B streptococcal disease: revised guidelines from CDC, 2010. MMWR. 2010;59(RR-10):1-23.

6. Melin P, Efstratiou A. Group B streptococcal epidemiology and vaccine needs in developed countries. Vaccine. 2013;31:D31-D42.

7. Bakhtiari R, Soltan Dallal MM, Mehrabadi JF, et al. Evaluation of culture and PCR methods for diagnosis of group B streptococcus carriage in Iranian pregnant women. Iran J Public Health. 2012;41(3):65-70.

8. Honest H, Sharma S, Khan KS. Rapid tests for group B streptococcus colonization in laboring women: a systematic review. Pediatrics. 2006; 117:1055-1066.

9. Håkansson S, Kallén K, Bullarbo M, et al. Real-time PCR-assay in the delivery suite for determination of group B streptococcal colonization in a setting with risk-based antibiotic prophylaxis. J Matern Fetal Neonatal Med. 2014;27(4):328-332.

10. Smith JM, Rexroth JA, Chaffin DG, et al. Serotyping group B streptococci in a small community hospital: an analysis of distribution and site of isolation. Infect Dis Obstet Gynecol. 2002;10:165-169.

11. Clinical and Laboratory Standards Institute. Performance standards for antimicrobial susceptibility testing, twenty-third informational supplement (M100-S23). CLSI, Wayne, PA, USA; 2013.

12. Ke D, Menard C, Picard FJ, et al. Development of conventional and realtime PCR assays for the rapid detection of group B streptococci. Clin Chem. 2000;46:324-331.

13. Uh Y, Jang IH, Hwang GY, et al. Serotypes and genotypes of erythromycin-resistant group B streptococci in Korea. J Clin Microbiol. 2004;42(7):3306-3308.

14. Wali IE, Sorour AE, d Abdalla MAH. Assessment of different methods for detection of group B streptococci carriage among pregnant females. EJMM. 2007;16(4):593-598.

15. Fang and Hedin. Rapid screening and identification of methicillinresistant Staphylococcus aureus from clinical samples by selective-broth and real-time PCR assay. J Clin Microbiol. 2003;41(7):2894-2899.

16. Bergeron MG, Gagnon M, Ke D, et al. Rapid detection of group B streptococci in pregnant women at delivery. N Engl J Med. 2000;343:175179.

17. Rallu F, Barriga P, Scrivo C, et al. Sensitivities of antigen detection and PCR assays greatly increased compared to that of the standard culture method for screening for group B streptococcus carriage in pregnant women. J Clin Microbiol. 2006;44(3):725-728.

18. Verani JR, Schrag SJ. Group B streptococcal disease in infants: progress in prevention and continued challenges. Clin Perinatol. 2010;37:375-392.

19. El Aila NA, Tency I, Claeys G, et al. Genotyping of Streptococcus agalactiae (group B streptococci) isolated from vaginal and rectal swabs of women at 35-37 weeks of pregnancy. BMC Infect Dis. 2009;9:153-163.

20. Kulkarni AA, Pawar SG, Dharmadhikari CA, et al. Colonization of pregnant women and their newborn infants with group B streptococci. Indian J Med Microbil. 2001;19(2):1-4.

21. Barcaite E, Bartusevicius A, Tameliene R, et al. Prevalence of maternal group B streptococcal colonization in European countries. Acta Obstetricia et Gynecologica Scandinavica. 2008;87(3):260-271.
22. Kovavisarach E, Ying WS, Kanjanahareutai S. Risk factors related to group B streptococcal colonization in pregnant women in labor. $\mathrm{J} \mathrm{Med}$ Assoc Thai. 2007;90:1287-1292.

23. Atkins KL, Atkinson RM, Shanks A, et al. Evaluation of Polymerase chain reaction for group B streptococcus detection using an improved culture method. Obstet Gynecol. 2006;108(3 Pt 1):488-491.

24. Jahromi BN, Poorarian S, Poorbarfehee S. The Prevalence and adverse effects of group B streptococcal colonization during pregnancy. Arch Iranian Med. 2008;11(6):654-657.

25. Dechen TC, Sumit K, Ranabir. Correlates of vaginal colonization with group B streptococci among pregnant women. J Global Infect Dis. 2010;2:236-241.

26. Costa AL, Lamy FF, Chein MB, et al. Prevalence of colonization by group B streptococcus in pregnant women from a public maternity of Northwest region of Brazil. Rev Bras Ginecol Obstet. 2008;30:274-280.

27. ElSayed S, Gregson DB, Church DL. Comparison of direct selective versus nonselective agar media plus Lim broth enrichment for determination of group B streptococcus colonization status in pregnant women. Arch Pathol Lab Med. 2003;127:718-720.

28. Philipson EH, Palermino DA, Robinson A. Enhanced antenatal detection of group B streptococcus colonization. Obstet Gynecol. 1995;85:437-439.

29. Liu GY, Doran KS, Lawrence T, et al. Sword and shield: linked group B streptococcal $\beta$-hemolysin/cytolysin and carotenoid pigment function to subvert host phagocyte defense. Proc Natl Acad Sci USA. 2004;101:14491-14496.

30. Votava M, Tejkalova'M, Dra'bkova' M, et al. Use of GBS media for rapid detection of group B streptococci in vaginal and rectal swabs from women in labor. Eur J Clin Microbiol Infect Dis. 2001;20:120-122.

31. Edwards RK, Novak-Weekley SM, Koty PP, et al. Rapid group B streptococci screening using a real-time polymerase chain reaction assay. Obstet Gynecol. 2008;111:1335-1341.

32. Bergh k, Stoelhaug A, Loeseth k, et al. Detection of group B streptococci (GBS) in vaginal swabs using real-time PCR with TaqMan probe hybridization. Indian J Med Res. 2004;119:221-223.

33. Smith D, Perry JD, Laine L, et al. Comparison of BD GeneOhm real-time polymerase chain reaction with chromogenic and conventional culture methods for detection of group B streptococcus in clinical samples. Diagn Microbiol Infect Dis. 2008;61:369-372.

34. Block T, Munson E, Culver A, et al. Comparison of carrot broth- and selective Todd-Hewitt broth-enhanced PCR protocols for real-time detection of Streptococcus agalactiae in prenatal vaginal/anorectal specimens. J Clin Microbiol. 2008;46:3615-3620.

35. Munson E, Napierala M, Munson KL, et al. Temporal characterization of carrot broth-enhanced real-time PCR as an alternative means for rapid detection of Streptococcus agalactiae from prenatal anorectal and vaginal screenings. J Clin Microbiol. 2010;48(12):4495-4500.

36. Lefevre J, Hankins C, Pourreaux K, et al. Real-time PCR assays using internal controls for quantitation of HPV-16 and betaglobin DNA in cervicovaginal lavages. J Virol Methods. 2003;114:135-144.

37. Kimura K, Suzuki S, Wachino J, et al. First molecular characterization of group B streptococci with reduced penicillin susceptibility. Antimicrob. Agents Chemother. 2008;52:2890-2897.

38. Nagano N, Nagano Y, Toyama M, et al. Nosocomial spread of multiresistant group B streptococci with reduced penicillin susceptibility belonging to clonal complex 1. J Antimicrob Chemother. 2012;67:849856.

39. Schoening TE, Wagner J, Arvand M. Prevalence of erythromycin and clindamycin resistance among Streptococcus agalactiae isolates in Germany. Clin Microbiol Infect. 2005;11:579-582. 
40. Pinheiro S, Radhouani H, Coelho $\mathrm{C}$, et al. Prevalence and mechanisms of erythromycin resistance in Streptococcus agalactiae from healthy pregnant women. Microb Drug Resist. 2009;15:121-124.

41. Lee BK, Song YR, Kim MY, et al. Epidemiology of group B streptococcus in Korean pregnant women. Epidemiol Infect. 2010;138:292-298.

42. Grimwood K, Stone PR, Gosling IA, et al. Late antenatal carriage of group B streptococcus by New Zealand women. Aust $N Z$ J. Obstet. Gynecol. 2002;42:182-186.
43. Gibbs RS, Schrag S, Schuchat A. Perinatal infections due to group B streptococci. Obstet Gynecol. 2004;104:1062-1076.

44. Acikgoz ZC, Almayanlar E, Gamberzade S, et al. Macrolide resistance determinants of invasive and noninvasive GBS in a Turkish hospital. Antimicrobial agents and chemotherapy. 2004;48(4):1410-1412. 